\title{
Tales of the Anthropocene: A Spatial Narrative of the Environment
}

\author{
JULIE LARSEN \\ Syracuse University
}

The anthropocene, the new geological age, characterized by long-term climatic change and the commencement of significant human impact, is a result of industrialization, on the earth's geology and ecosystems. While architecture itself cannot provide universal solutions, as urgent environmental threats become increasingly more visible, the role of architecture can help facilitate the understanding of those effects of climate change on our current culture. Architecture, as a material practice, can provide future speculations of environmental shifts on the earth through materialization, visualization, and speculation. Using a series of advanced design studios and seminar work as case studies, the paper aims to address the way in which architects can bring more awareness to significant environmental concerns through visual provocations. Using architectural tools as design research with mappings, drawings, and time-based imagery, visualizations materialize the potential risks and outcomes of changes to ecologies around the globe. With tales of the anthropocene, the focus on form, materiality and spatial narrative reflects how architecture can play a critical role in revealing the impact and effects of human activity on the environment.

\section{INTRODUCTION}

For researchers and scientists, an unresolved challenge is measuring impact and deciphering the way we can measure the human imprint on the geologic record and how it can be made visible. Architecture, as a visible object, can foster social awareness of environmental concerns that are both cultural and aesthetic problems as much as technological and performative ones. And due to the scale of climate change, the environment is 'neither inherently invisible or inherently visible; it is, like all other objects, made visible' (Rudiak-Gould, 2013). Some of the most significant effects to our cities and the environment are 'not registered as images' and to begin to 'map this unmappable territory, the conventions of representation itself need to be rethought. If architecture is to work beyond the level of image it needs to invent new tools [and] to work effectively within the immaterial networks and systems' (Allen). In regards to these rapidly changing climates, instability, and alteration over time is a challenge to reconcile and visualize. 'The city today is a place where visible and invisible streams of information, capital, and subjects interact in complex formations. In order to describe or to intervene in this new field architects need representational techniques that engage time and change, shifting scales, mobile points of view, and multiple programs. To map this complexity, some measure of control may have to be relinquished'
(Allen, 2000). It is this measure of control which was the aim to visualize the rapid changes of particular biomes around the planet. This initial design research was used to define design interventions that relinquish some, if not all, control to the architectual outcome where the spatial narrative reflects significant changes over time, whether that's through changes in the environment, materiality, or form.

\section{INVISIBLE MADE VISIBLE}

In the spirit of the Whole Earth Catalog (WEC), the first publication on the whole earth system, students were in search of other forms of visualization to describe the effects of climate change in the Anthropocene Era. Similar to the WEC, students studied particular regions and climates to analyze and visualize human activity that caused significant change to the environment. Through new online tools, aerial image captures generated initial narratives as a point of departure for the design research. Students were asked to do a series of image captures of a particular region within a biome and extract the images from Planet.com, a company that launched 100 s of 'dove satellites', which are very small earth imaging satellites comprised as a constellation that 'line scan' the planet and capture the entire surface of the Earth every day (Planet, 2018). The students extracted a series of images spanning many years from Planet.com and converted them to a Planet.com 'Story' or a digital .gif that enables one to visibly track changes to a particular site. Typically, within just a few years, one can see remarkable change within a short period of time. With these image captures the students were asked to make legible a significant visual and physical change in a particular region that traditionally Google Earth does not capture because its image captures are updated, on average, only every six months. They presented this research digitally through time-lapse GIF-files of the existing conditions of their chosen 'site' of inquiry and the surrounding landscape. The student's time-lapse images reveal rapid man-made changes impacting the land, that through traditional site analysis, would remain invisible.

\section{MEASURING IMPACT}

As mentioned, the challenge is not only in measuring obvious impact through physical changes but also in deciphering a way to measure the invisible human imprint and how it can be made visible. The extracted images from Planet.com were used to measure and comprehend the impact of human activity (such as mining, oil drilling, or deforestation, etc.) on a particular region on earth. The aim was to use the time-based 

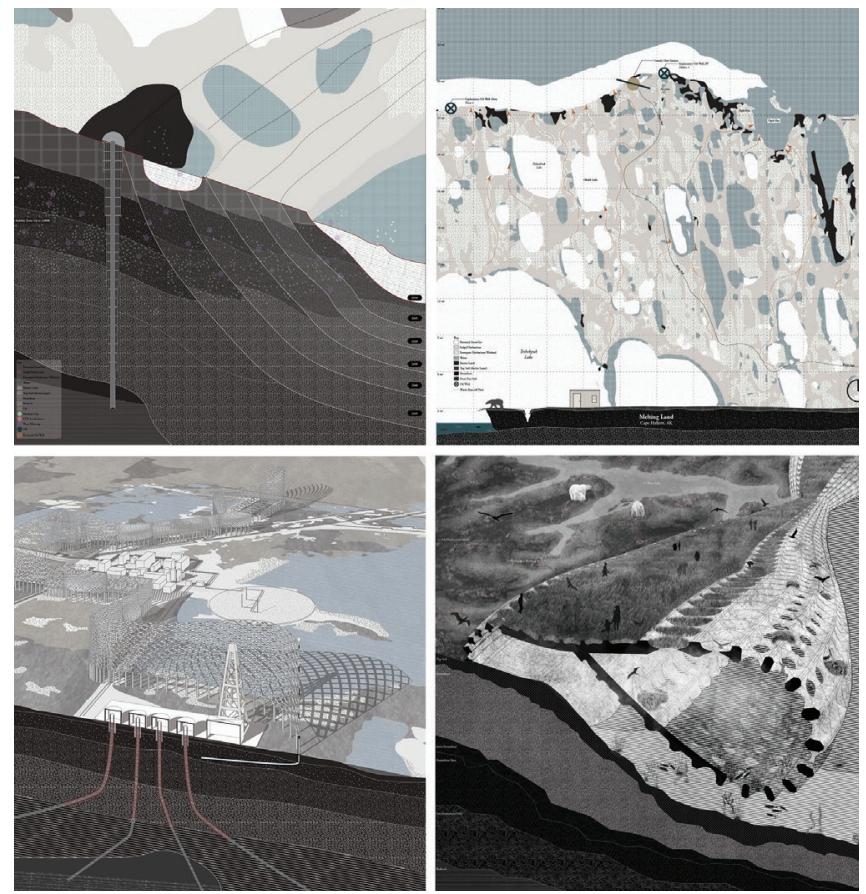

Figure 1. From upper left, clockwise: Section of Alaska permafrost melting due to oil drilling, Mapping of Alaska permafrost and cliff edge deterioration due to softer ground, Wave break and energy generator as competitive solution to oil, and refreezing of the ground through piped surfaces that envelope oil drilling facilities (drawings by Rasan Taher and Davey Bullard).

imagery to visualize both the 'visible' and 'invisible' impacts within in a particular site of a biome. Those impacts were visualized in a series of mappings based on the original aerials of the sites. The visible variables were comprehensible as evident physical changes to the landscape, such as water fluctuations, vegetative or land deterioration, flooding, etc. But often these are indications for invisible variables that were not easily discernible by looking at images, such as pollution, habitat loss, depletion of resources, or poor air quality. Students were asked to use drawings to convey how sites were altered over time due to the impact of both those visible and invisible factors. For example, in one of the project's time-lapse images of La Pampa, Peru, it revealed a series of gold mines destroying the Amazon landscape over a condensed period of time from January 2016 to January 2017. Using topographic lines from the changing terrain, as well as sections and images from the urban to the human scale, the narrative for this project began by showing how much destruction the La Pampa gold mine had caused due to the rising worth of gold. After studying the aerial photography and researching the region beyond the immediate site, the students discovered how mercury was used to extract the limited amount of gold from the slurry created, which poisons the miners and the area surrounding the mines. This research led the students to create a new narrative of the site where carbon filters line the riverbed of the adjacent town in the aftermath of the mining in the hopes that the water can become clean enough for future fishing. A second project shows a time-lapse revealing the effects of permafrost in the
Alaskan region of the North Slope (Figure 1a, b). The mapping describes the causes of melting permafrost on the North Slope of Alaska, ranging from climate change to oil drilling, which are variables in the region's drastic coastal erosion, melting permafrost, and subsequent release of methane into the enviroment. The students proposed two spatial narratives which take two alternative departures on how to respond to this crisis. The first narrative takes a more opportuntistic approach that begins with speculation on other renewable energy sources as an alternative to oil to prevent future expansion of the industry in the region. The second narrative accepts that oil is there to stay and depicts a scenario in which we need to accept this new industrialized landscape and respond to present threats that will persist in the wake of continued drilling. A third project reveals that the Mediterranean Sea is one of the most polluted seas in the world due to the amount of trash, and specifically plastics, dumped into to the sea on a yearly basis. The project studied water currents and shipping routes in the Mediterranean as an existing resource to render the volume of waste more visible (Figure 2a). This research translated to a speculation on how to use existing maritime infrastructure to circumvent the continual pollution of the water.

Lastly, through research on Indonesia students discovered that the country has the fastest rate of deforestation in the world. The students explored many locations within Indonesia, from the West Kalimantan region to the Ciliwung River to Jakarta, all areas that were previously surrounded by forested watershed that would store and stabilize soil but are now regions flooded on a regular basis due to deforestation and, in turn, destabilized ground and erosion (Figure 3a, d). The research on Indonesia was an opportunity to create a new narrative generated around wood that is now a significantly reduced resource. This offers a new narrative to a region's struggle to overcome economic and political divisions reaking havoc on the environment throughout the country. For example, the West Kalimantan region of Indonesia was once a place where vegetation flourished uncontrolled and untouched by humans but is disappearing due to economic needs for new palm oil plantations (Figure 3d, e). Currently, it is a forest that has been substantially cleared, compromising local species of flora and fauna. Even with government efforts to preserve the forest, there is no alternative than for animal species to migrate elsewhere. To avoid further expansion of the palm oil plantations, the students used drawings to speculate on whether an architectural intervention could permanently define the existing edge between forest and agriculture (Figure $3 f$ ).

\section{CONSTRUCTING THE ANTHROPOCENE}

The early mappings of various biomes led to research of technology, materials, and systems to develop more responsive interventions to environmental issues. Responsive structures were used as a speculative and open-ended response; therefore, they were not limited in scale or occupation but aimed to inhabit a particular environment in order to visualize the 

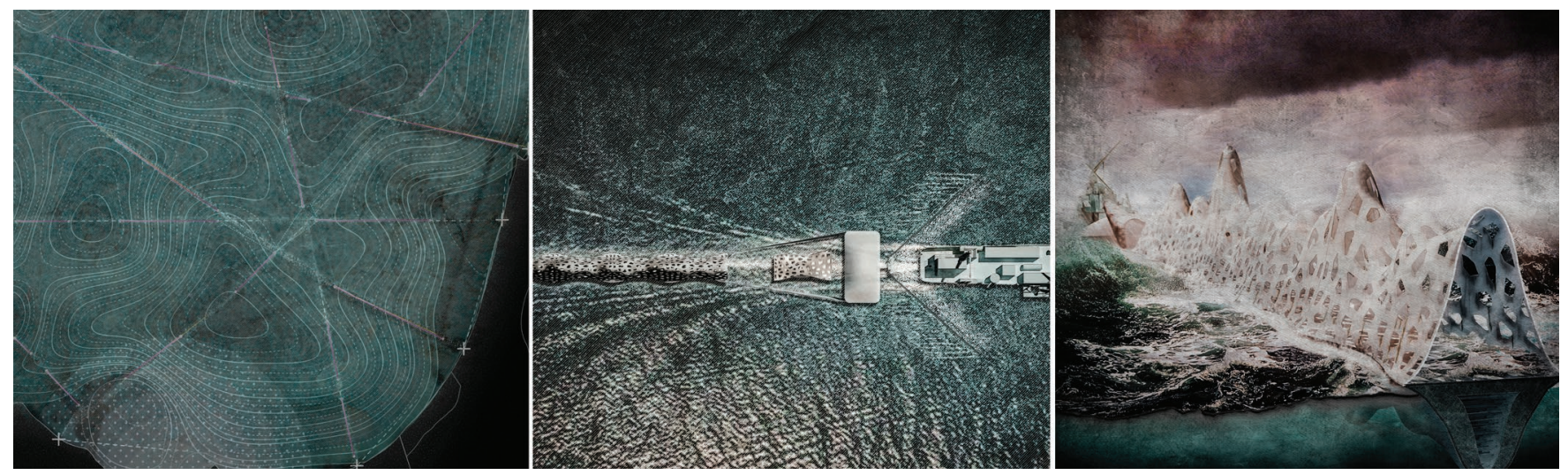

Figure 2. Left, Mapping of shipping lines, water currents, and movement of trash and plastic in the Mediterranean Sea; center, Speculation on collecting and harvesting plastics with existing barges and ships; right, Inhabitable barrier floating in the Mediterranean (drawings by Rodrigo Lopez).

impact of climate change. The following work was developed as iterative and aggregated surfaces or structures that respond to local conditions, using low or high-tech solutions. The constructed structures reflect variations within a given system, such as wind patterns, water flow, condensation, growth, or rates of filtration, that adapt and respond over time as either a daily, seasonal or yearly fluctuation. The study of specific materials, such as wood, concrete, or recycled plastic was explored through various tools and methodologies of stacking, interlocking, or aggregating to begin to materialize the issues of climate change in their respective regions. For example, in Indonesia, exposing an excessive amount of interlocking wood structures is used for potentially miles of linear sites to sequester increasing greenhouse gas emissions within the surface of the wood (Figure 3b, c). A second project researched air pollution in Monterrey, Mexico and, in turn, studied concrete that is capable of sequestering $\mathrm{CO} 2$ that would, otherwise, be absorbed into the atmosphere (Biello, 2008). With the project researching the Mediterranean Sea, trash and plastic are recycled into a specific resin that was developed by Physicist and engineer Klaus Lackner, and can be configured into floatable structures that capture $\mathrm{CO} 2$ from the sea at 1000 times higher efficiency than an actual tree (Cantieri, 2016) (Figure 2c).

\section{THE TALES OF THE ANTHROPOCENE}

While the work so far revealed the issues and investigated possible technical solutions, in this next step, the students developed spatial and material narratives, or 'tales', from their observations on the anthropocene. In developing a 'tale of the anthropocene,' the following projects define speculation and intervention through the notion that 'stable objects' are obsolete and replaced with objects that are more emergent and responsive to their environment. The aim was for the projects to adapt to the changing dynamic quality of the region's climatic conditions. The following projects shift perception of the Anthropocene to propose radical gestures of renewed equality between human as well as non-human actors, using materiality, form, and spatial narrative to speculate on future scenarios in sites drastically affected by human actors.

\section{TALE \#1 - JAKARTA, INDONESIA}

It has been estimated that approximately 32 gigatons of carbon dioxide are produced each year (IEA, 2017). Jakarta, the world's second largest megacity, experiences a number of challenges, including air pollution due to high $\mathrm{CO} 2$ emissions produced by the increasing volume of cars. The absence of forest in this urban area has reduced sequestration of increasing greenhouse gas emissions. The tale begins with a speculative vertical structure in the center of Jakarta that soaks up $\mathrm{CO} 2$ emissions through the exposed wood (Figure $3 c)$. As one reaches the fifteenth floor, past the undulating walls and through plywood screens, one looks out to the city, and witnesses dozens of vertical wood structures breaking through the misty haze as a series of clouded silhouettes in the skyline. These timber structures are comprised of thousands of thresholds and interlocking connections that allow contaminated air to be sequestered in the fibers of the wood (Figure 5). Over time, this air is stored, absorbed and visibly tarnishing the material, a coding of the effects of the material on its surrounding environment.

\section{TALE \#2 - NORTH SLOPE, ALASKA, USA}

This tale begins with the effects of permafrost and the release of methane which is softening the ground and having a profound impact on the eroding coastline of the Alaskan North Slope (Figure 1a, b). The earlier mapping described the causes of melting permafrost due to local oil drilling and warmer air temperatures due to climate change. The melting permafrost layer causes environmental issues due to greenhouse gases, such as Methane, that has been stored deep in the layer of ice for thousands of years as well as large numbers of toxic mercury released into the atmosphere as the layers continue to melt, causing coastal erosion in the region. The first proposal is a tale for the North Slope to reverse the melting of the permafrost layer and to stabilize the ground in critical areas of oil drilling, the project refreezes the ground by pumping liquid carbon dioxide into pressurized pipes (Figure 1c). The liquid has a very low evaporation point and rises above ground through the piped structure into radiators where the liquid 

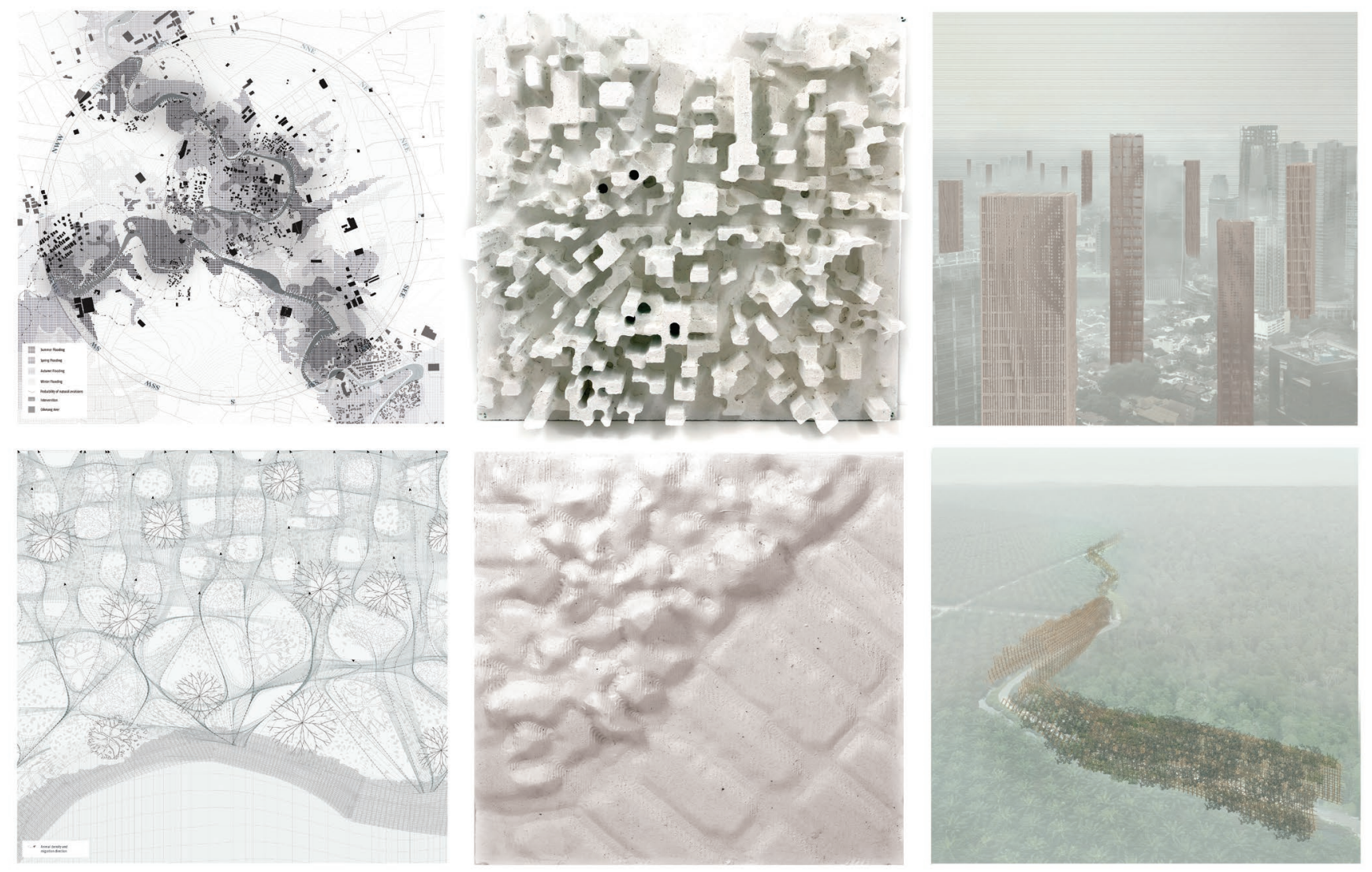

Figure 3. Top left, Site mapping of Ciliwung River, Indonesia; top center, CNC milled surface of Jakarta; top right, wood towers to sequester CO2 emissions; bottom left, Site mapping of migration patterns in West Kalimantan, Indonesia; bottom center, CNC milled surface depicting edge between the forest and plantation; bottom right, time-based .gif showing inhabited structure at edge of forest (drawings by Shaguni Gupta and Andrea Dominguez).

condensates as the heat is released into the atmosphere. The cycle continues as long as the temperature is colder above ground. Over time, this spatial narrative keeps the ground frozen under critical infrastructures to prevent potential oil spills and reduce erosion. The second proposal, or an alternative tale, for the North Slope, is a more opportunistic approach that speculates on alternative energy resources to oil drilling with a wave break that that serves as a protective barrier while stabilizing the ground. The chain of wave breaks would create passive energy by harnessing the power of waves. The surfaces of the wave energy harvesting encourage wildlife, both above and below the water to foster harbor species whose habitats are being washed away by coastal erosion.

\section{TALE \#3 - WEST KALIMANTAN, INDONESIA}

This tale begins with the intertwined concerns of visualizing climate change and the spatializing effects that deforestation has on landscapes through materiality. Deforestation, as a factor in the transformation of land, contributes to the cutting of fifteen billion trees each year and is tied to illegal logging areas which are not managed to ensure forest regeneration. The visual narrative begins with an open structure made with manufactured timber that straddles between the oil plantation and the forest. The overgrown structure is indistinguishable as its original form over time as it becomes inhabited by wildlife. One can barely distinguish the timber members from those of the tree branches as they become tangled and morphed into one object (Figure 4). It is the original edge condition in West Kalimantan, Indonesia where vegetation once flourished uncontrolled, untouched by humans but has now disappeared because of economic needs to expand palm oil plantations. The forest was substantially cleared, compromising local species, flora and fauna, that has left no other alternative than for animal species to migrate, even with government efforts to preserve the forest. Where the structure is denser and enclosed, it is occupied by humans and used as storage and equipment for the plantation. The new narrative is one of significantly low stakes but has the potential to have profound impact as an inexpensive, infrastructural element. By simply placing a framed, wood structure adjacent to the remaining forest, the boundary is set and the continuation of the plantations becomes significantly challenging to expand beyond a strong, visible barrier. The barrier becomes a visible icon in the region that can not be ignored. The structure takes the invisible economic demands and merges them with the visible and physical alterations to the landscape and puts it on display as a datum that should be preserved. 


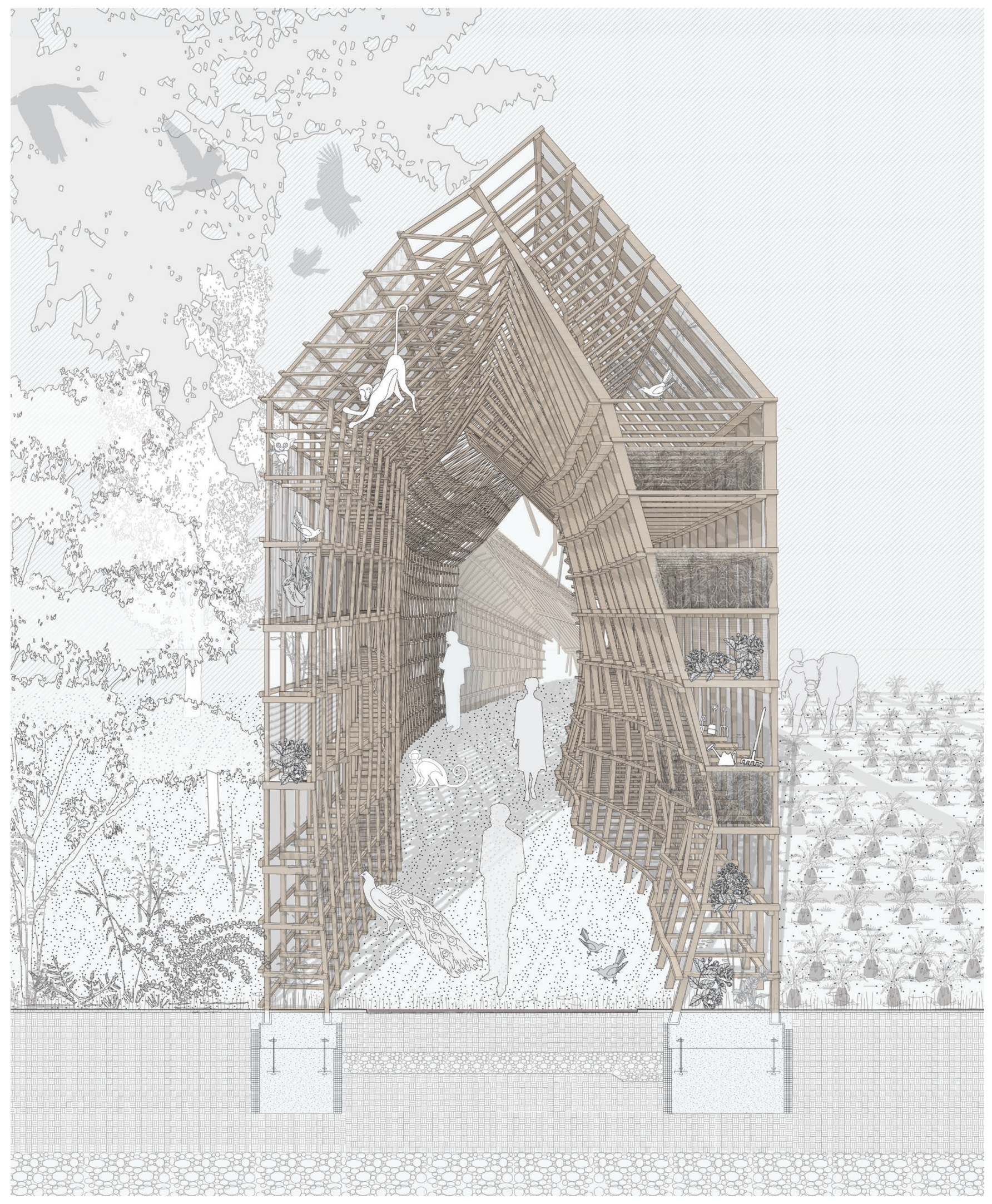

Figure 4. Inhabited structure in West Kalimantan, Indonesia (drawing by Shaguni Gupta and Andrea Dominguez). 


\section{CONCLUSION}

The potential for architecture, as a material practice, to play a pivotal role in how we begin to comprehend the impact of climate change, lies in how we interrogate the Earth through materiality, tools and technology as a way to render climatic shifts in a more visual and impactful way. If the Anthropocene is the result of human creation and destruction then architectural speculations can begin to see past the crisis as merely catastrophic and visualize an alternative reality.

And with new technology available that provides real-time data and visual information, design research can extend beyond mapping and speculation to discover new material and spatial narratives that reach beyond data, information and charts. The discussed projects each write their own Tale of the Anthropocene to assemble a narrative that brings together the larger impact of climate change together with a more local engagement at the scale of materiality, space and form. The Tales of the Anthropocene begin to visualize a spatial narrative to foster public engagement but also to understand that visualizing these calamitous realities are as much an aesthetic and spatial problem as a technological one.

\section{REFERENCES}

Allen, Stan, and Diana Agrest. Practice: Architecture, Technique and Representation. G+B Arts International, 2000, 31-45.

Biello, David. "Cement from CO2: A Concrete Cure for Global Warming?" Scientific American, August 7, 2008. www.scientificamerican.com/article/ cement-from-carbon-dioxide/.

Cantieri, Janice. "Artificial Trees Could Offset Carbon Dioxide Emissions." Climate Change. climatechange.medill.northwestern.edu/2016/11/29/ artificial-trees-might-be-needed-to-offset-carbon-dioxide-emissions/.

International Energy Agency (IEA). “Global Carbon Dioxide Emissions, 1980-2016." March 17, 2017. www.iea.org/newsroom/energysnapshots/global-carbon-dioxideemissions-198-2016.html.

Mirzoeff, Nicholas. "Visualizing the Anthropocene." Public Culture 26, no. 2 (2014): 213-232. doi:10.1215/08992363-2392039.

Planet official website, “Approach” page. Accessed May 7, 2018. www.planet.com/ company/approach/.

Rudiak-Gould, Peter. "We Have Seen It with Our Own Eyes': Why We Disagree About Climate Change Visiblity." Weather Climate \& Society 5 (2013): 128.

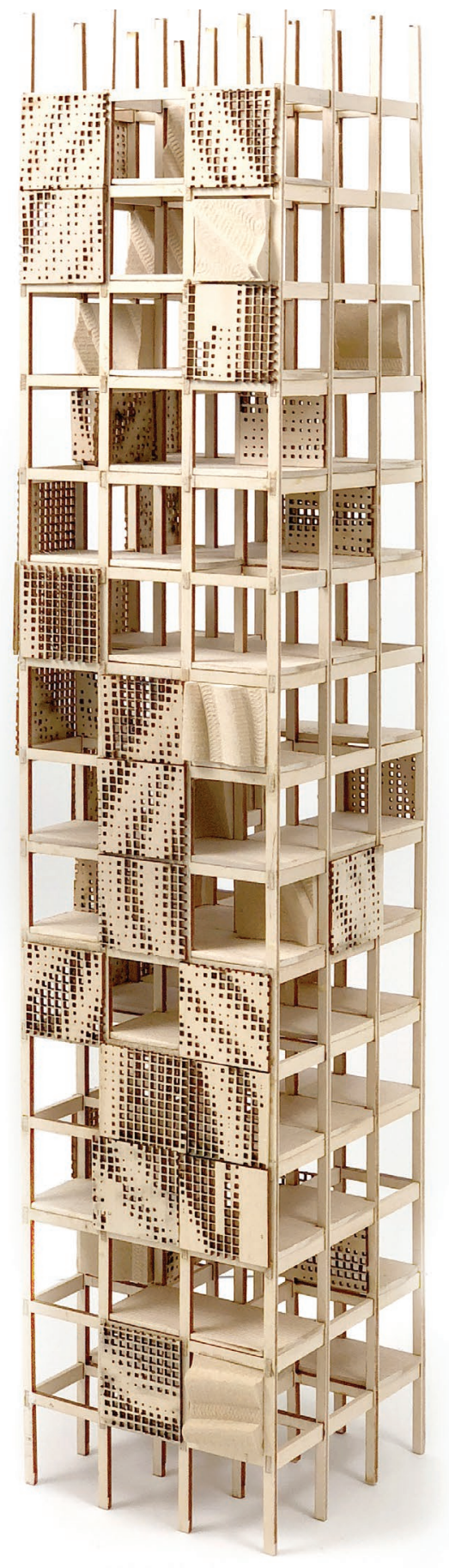

Figure 5. Wood towers to sequester CO2 emissions in Jakarta, Indonesia (model by Shaguni Gupta and Andrea Dominguez). 